\title{
Pay-Load Estimation of a 2 DOF Flexible Link Robot
}

\author{
N. K. Poulsen ${ }^{1}$ and O. Ravn ${ }^{2}$ \\ ${ }^{1}$ Informatics and Mathmatical Modelling, Technical University of Den- \\ mark, nkp@imm.dtu.dk; ${ }^{2}$ Ørsted•DTU, Technical University of Den- \\ mark., or@oersted.dtu.dk.
}

\begin{abstract}
The paper presents a new method for online identification of pay-loads for a two-link flexible robot. The method benefits from the close correspondence between parameters of a discrete-time model represented by means of the Delta-Operator, and those of the underlying continuous-time model. Although the applied principle might be general in nature, the paper is applied to the well-known problem of identifying a pay-load of a moving flexible robot. The presented method benefits from the close correspondence with the continuous-time representation to allow a scalar and implicit adaptive technique which based on flexibility measurements leads to the online estimation of the pay-load.
\end{abstract}

Keywords: Flexible Link Robot; Delta-Operator; System Identification; Parameter Estimation; Adaptive Control.

\section{Introduction}

Flexible robot systems are motivated by a desire for better arm-weight to pay-load ratios, shorter travel-times and lower energy consumption.

The desire for high-performance manipulators and the benefits offered by a light-weight flexible link capable of maneuvering large pay-loads have lead to analysis of the behavior of the dynamics in which flexibility is the essential issue. The high-performance requirements will inevitably pro- 
duce designs that during operation will excite vibrations in the manipulator structure.

The aim of the controller is to suppress the structural vibration while in addition to minimize the cycle time of the manipulator system. In this work we will use a model-based controller in order to mitigate the first harmonics. However, changes in pay-load degrade the model and consequently the performance of the control system, unless some sort of adaptation or gain-scheduling is taken into account to estimate these effects.

In order to investigate different aspects of control of flexible links robot configurations an experimental setup has been made and a simulation model has been developed. The setup consists of two very flexible links with two actuators located in the joints. In this work the links are moving in the horizontal plane making gravity ignorable. The actuators are DCmotors with a sufficient gear ratio and tachometers making an analog velocity feedback feasible. Apart from the tachometers there are also two sensors on the setup, a potentiometer in each joint enabling a measurement of the position of the joint and a number of strain gauges located on each link enabling the measurement of the deflection of the link.

The literature contains experimental results featuring gravity compensation is presented for a double link robot with a flexible forearm. But like other references, practical algorithms seem to ignore the fact that one could construct an adaptation technique that directly gains insight to the pay-load parameter. This is topic for the present work. By deducting a linear statespace model describing the pay-load parameter's influence in the continuous-time model, it is possible to apply a Delta-Operator technique for estimation of this parameter in discrete-time.

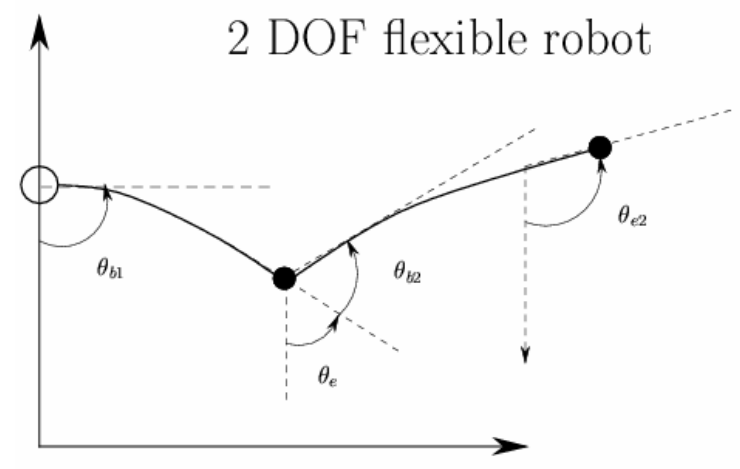

Fig. 1. The flexible robot system consists of two flexible links that are coupled together through actuator 2 . 


\section{Design model of the system}

The flexible manipulator system studied here, see Fig 1, carries a pay-load, $\mathrm{m}_{\mathrm{p}}$, at its tip and moves in the horizontal plane. The active degrees of freedom are the two rotational angles $\theta_{1}$ and $\theta_{2}$.

In literature the equations of motions are commonly modeled by either a Finite Element Method (see. e.g. [1]) or the Eigenvalue Method (see e.g. [2]). As the latter method is normally considered more accurate when only a limited number of modes are included, cf. [3], the following description is be based on this approach, cf. [4] .

The model of the flexible link robot consists of four parts; namely the models for the two actuators and the two arms. The dynamics of the flexible arms can be described by a PDE which can be transferred into a ODE by using the method of separation of variable. In that case the deflection, $\omega_{j}(x, t), j=1,2$, of the arms is approximated by a finite sum of contributions

$$
\omega_{j}(x, t)=\sum_{i=1}^{n} \phi_{j i}(x) q_{i j}(t)
$$

where $\phi_{\mathrm{ji}}(\mathrm{x})$ and $\phi_{\mathrm{ji}} \mathrm{q}(\mathrm{t})$ are the normal and harmonic function of mode $\mathrm{i}$ and $\operatorname{arm} \mathrm{j}$, respectively.

\subsection{Equations of Motion in Compact Form}

If the actuator equations are used for obtaining the angular accelerations the four main equations can be written in a more compact form Introducing the notation:

$$
\underline{q}=\left[q_{11}, \ldots q_{1 n}, q_{21}, \ldots q_{2 n}\right]^{T}, u=\left[\begin{array}{l}
u_{1} \\
u_{2}
\end{array}\right], \underline{\theta}=\left[\begin{array}{l}
\theta_{1} \\
\theta_{2}
\end{array}\right]
$$

The the descripton of the flexibility can be linearized and be brought into the following compact form:

$$
\underline{\ddot{q}}=M_{1} \underline{q}+M_{2} \underline{\dot{q}}+M_{3} \underline{q}+M_{4} \underline{u}+M_{5} \underline{\dot{u}}
$$

Where the matrices $M_{2} M_{3}$ and $M_{5}$ are linearly dependant on $m_{p}$. Notice the matrices depend on the linearization point. In this case the matrices depend only on $\mathrm{q}_{2}$. Also notice the angular acceleration q occurs on both sides of the i equation.

Also the actuator dynamics can be written in a compact form. 


$$
\dot{\theta}=M_{6} \underline{q}+M_{7} u
$$

Now the compact description in (3) and (4) is to be transformed into a state space description. It is possible to establish the following linear dependencies of $\mathrm{m}_{\mathrm{p}}$

$$
\begin{aligned}
& M_{2}=M_{2}^{0}+m_{p} M_{2}^{m} \\
& M_{3}=M_{3}^{0}+m_{p} M_{3}^{m} \\
& M_{5}=M_{5}^{0}+m_{p} M_{5}^{m}
\end{aligned}
$$

while $\mathrm{M}_{1}, \mathrm{M}_{4} \mathrm{M}_{6}$ and $\mathrm{M}_{7}$ are independent of $\mathrm{m}_{\mathrm{p}}$. If we define:

$$
\begin{aligned}
\underline{A}^{0}= & {\left[\begin{array}{ccc}
0 & M_{6} & 0 \\
0 & 0 & I \\
0 & \Lambda M_{1} & \Lambda M_{2}^{0}
\end{array}\right] \quad \underline{A}^{m}=\left[\begin{array}{ccc}
0 & 0 & 0 \\
0 & 0 & 0 \\
0 & 0 & \Lambda M_{2}^{m}
\end{array}\right] } \\
& \underline{B}^{0}=\left[\begin{array}{c}
M_{7} \\
\Lambda M_{5} \\
\underline{B}_{3}^{0}
\end{array}\right] \quad \underline{B}^{m}=\left[\begin{array}{c}
0 \\
\Lambda M_{5}^{m} \\
\Lambda M_{2}^{m} \Lambda M_{5}^{0}+\Lambda M_{2}^{0} \Lambda M_{5}^{m}
\end{array}\right]
\end{aligned}
$$

where

$$
\underline{B}_{3}^{0}=\Lambda M_{2}^{0} \Lambda M_{5}^{0}+m_{p}^{2} \Lambda M_{2}^{m} \Lambda M_{5}^{m}+\Lambda M_{4}
$$

then the system is described on a state space form.

The measurement system consists of two potentiometers and four strain gauges. The potentiometers give measurements of the link angles $\theta_{\mathrm{b} 1}$ and $\theta_{\mathrm{b} 2}$, whereas the strain gauges are located tactically on the links in order to give measurements of the deflections i.e. q.

\section{Simulation experiment}

In order to investigate the properties of the algorithm there have been performed a series of simulations. One simulation is described in the following. 
The simulation (and the design) model of the deflection is a two mode approximation. The reference signals are square waves for the link angles and the signals are shown in Figure 2. The sampling period was chosen to $\mathrm{T}=0.01 \mathrm{sec}$.
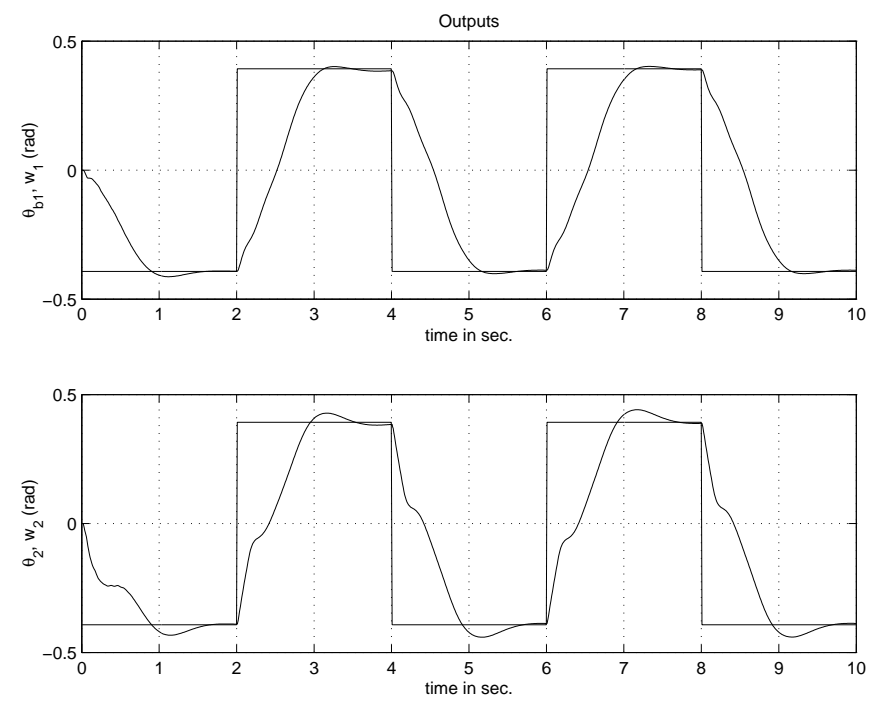

Fig. 2. Angular positions $\theta_{\mathrm{b} 1}$ and $\theta_{\mathrm{b} 2}$ presented as measured outputs (solid line) and as reference trajectories (dashed line).
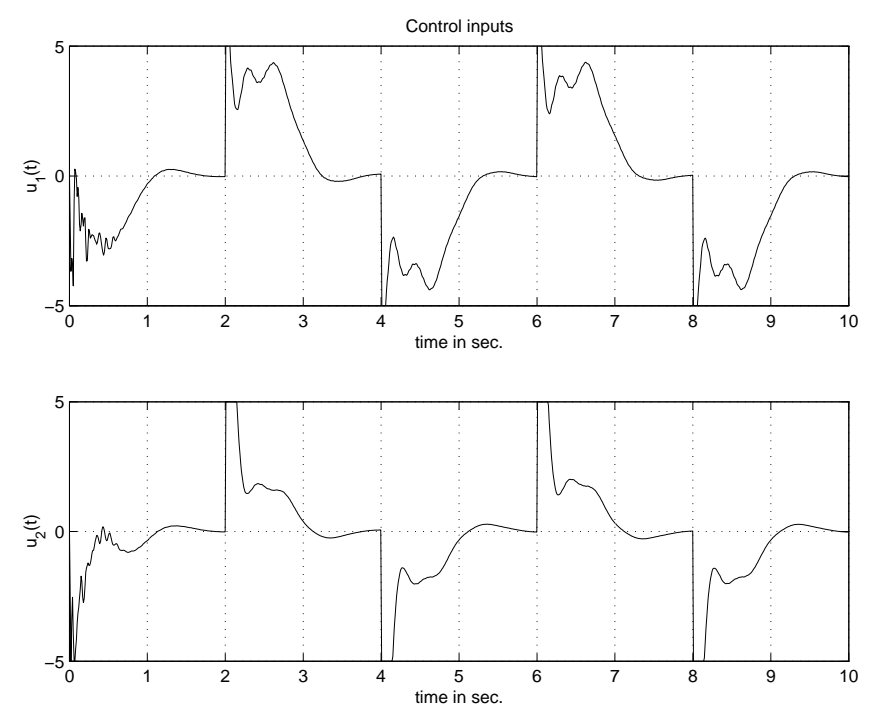

Fig. 3. Control inputs $\mathrm{u}_{1}$ and $\mathrm{u}_{2}$ as functions of time. 

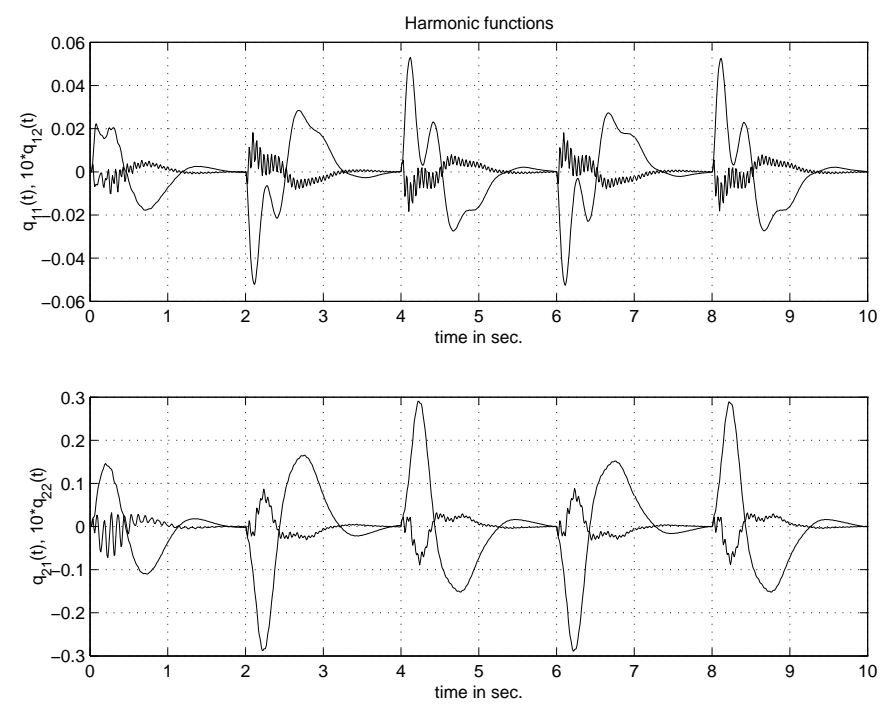

Fig. 4. Harmonic time-functions $\mathrm{q}_{\mathrm{i} 1}$ and $10 \mathrm{q}_{\mathrm{i} 2}$ for each of the beams, $\mathrm{i}=1,2$ presented as functions of time

The controller is a state space LQG controller which parameters are iterated one step per sample in order to follow the adaptation on $m_{p}$. This would asymptotically producing the optimal feed-back gain based on the loss weights

$$
\mathrm{Q}_{1}=\mathrm{C}^{\mathrm{T}} \mathrm{C} \quad \mathrm{Q}_{2}=0.005 \mathrm{I}
$$

The control is based on the estimated state of the system which are obtained by means of the predictive Kalman filter with

$$
\mathrm{R}_{1}=10^{-4} \mathrm{I} \quad \mathrm{R}_{2}=10^{-4} \mathrm{I}
$$

where $R_{1}, R_{2}$ denote respectively the process and the measurement covariance matrices. In spite of the deterministic simulation, the process noise covariance is needed in order to compensate for the unknown pay load mass in the model. 

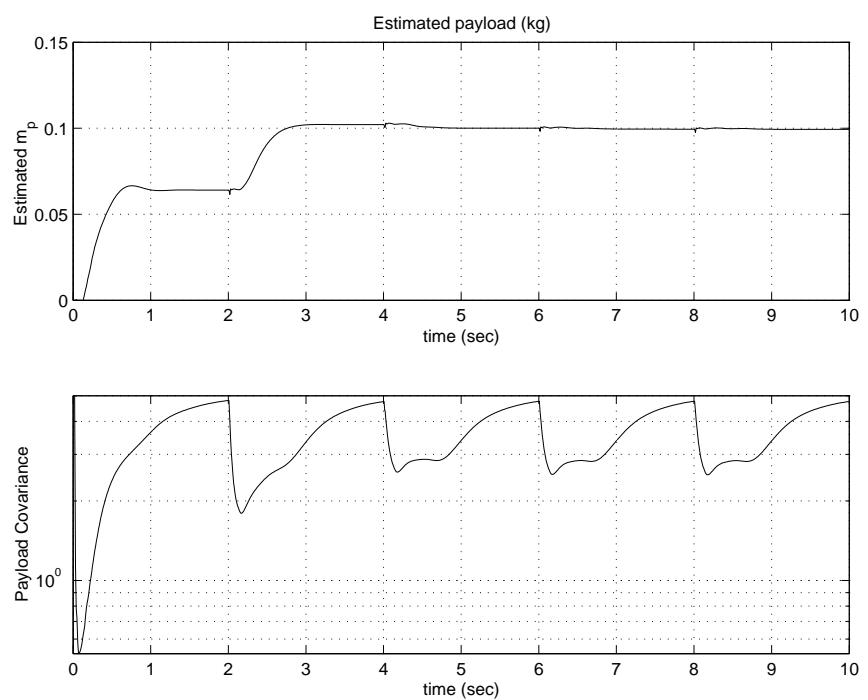

Fig. 5. Estimated pay-load $m_{p}(t)$ and variance of estimate in a closed-loop experiment.

The payload mass was estimated using the following parameters

$$
\alpha_{0}=0.1 \quad \alpha_{1}=10 \quad \mathrm{P}_{\mathrm{o}}=10
$$

The value of $m_{p}=0.1 \mathrm{~kg}$ can be compared to e.g. the mass of the lower arm, $\mathrm{m}_{12}=0.133 \mathrm{~kg}$, saying that the manipulator is heavily loaded. A better performance can be seen after the second step reflecting that $\mathrm{m}_{\mathrm{p}}$ is almost estimated after 3 seconds, see Figure 5. From here it also appears that only when the set-points are varied new information is obtained.

\section{Conclusions}

This paper has presented a method for online identification of pay-loads for a two-link flexible robot. The method is based on a state-space model of the flexible link which has been transformed into the discrete time domain using the Delta-Operator. This enables a close correspondence between the parameters in the discrete-time model and the underlying continuous-time model. Due to the close correspondence, it is shown that both domain models can produce almost the same linearity with respect to a pay-load. This fact is used in a pay-load estimation technique. By simulation it is demonstrated that it is possible to identify a time-varying payload of a two link flexible robot during closed-loop control. 
$8 \quad$ N. K. Poulsen1 and O. Ravn2

\section{References}

1. Sakawa, Y., Matsuno F. and Fukushima S. (1985): Modelling and control of a Flexible arm. Journal of Robotic Systems, 2, 453-472.

2. Kruise L. (1990): Modelling and control of a flexible Beam and Robot Arm. Ph.D. Thesis, University of Twente

3. Baungaard, J.R. (1996): Modelling and Control of Flexible Robot Links. Ph.D. thesis. Ph.D. thesis. Department of Automation, The Technical University of Denmark.

4. Rostgaard M (1995): Modelling, Estimation and Control of Fast Sampled Dynamic Systems. Ph.D. theses, Department of Mathematical Modelling, The Technical University of Denmark

5. Caspersen, Morten Keller (2000): Control of a Flexible Link Robot. Master Thesis, Department of Automation, The Technical University of Denmark.

6. Luca, A.D. and Panzieri, S. (1994): An iterative scheme for learning gravity compensations in flexible robot arms. Automatica, 30(6), 993-1002.

7. M'Saad, M, Dugard, L. and Hammand, S. (1993): A suitable generalized predictive adaptive controller case study of a flexible arm. Automatica, 29(3), 589-608.

8. Ravn, O and Poulsen, N.K. (2004): Analysis and design environment for flexible manipulators. Chapter 19 in Tokhi, M.O. and Azad, A.K.M.: Flexible robot manipulators - modeling, simulation and control.

9. Rostgaard ,M, Poulsen, N.K. and Ravn, Ole (2001): Pay-load estimation of a 2DOF flexible link robot using a delta operator technique. IEEE Conference on Control Application, Mexico, (248-253).

10. Timoshenko, S., Young, D.H. and Weaver, J.W. (1974). Vibration problems in Engineering. John Wiley and Sons. 